\title{
Weak Solutions for a Second Order Dirichlet Boundary Value Problem on Time Scale
}

\author{
Wandong Lou \\ Department of Foundation, Shandong Yingcai University, Jinan, Shandong 250104, China \\ Correspondence should be addressed to Wandong Lou; louwdll@163.com
}

Received 7 July 2013; Revised 7 October 2013; Accepted 9 October 2013

Academic Editor: Jin Liang

Copyright (C) 2013 Wandong Lou. This is an open access article distributed under the Creative Commons Attribution License, which permits unrestricted use, distribution, and reproduction in any medium, provided the original work is properly cited.

We adopt the Leray-Schauder degree theory and critical point theory to consider a second order Dirichlet boundary value problem on time scales and obtain some existence theorems of weak solutions for the previous problem.

\section{Introduction}

In recent years, differential equations on time scales have been studied extensively in the literature. There have been many approaches to study the existence and multiplicity of solutions for differential equations on time scales. The variational method is, to the best of our knowledge, novel, and it may open a new approach to deal with nonlinear problems on time scales. For more details about recent development in the direction, we refer the reader to [1-12] and the references therein. The two books $[13,14]$ by Bohner and Peterson summarize some excellent results on time scales.

In $[1,2]$, the authors utilized variational techniques and critical point theory to derive some sufficient conditions for the existence of positive solutions for the following second order dynamic equation with Dirichlet boundary conditions:

$$
\begin{gathered}
-u^{\Delta \Delta}(t)=f\left(\sigma(t), u^{\sigma}(t)\right), \quad \Delta \text {-a.e. } t \in J, \\
u(a)=0=u(b) .
\end{gathered}
$$

In [8], Zhou and Li studied Sobolev's spaces on time scales, and given their properties, as applications, they presented variational methods and critical point theory to obtain the existence of solutions for the second order Hamiltonian systems on time scales as follows:

$$
\begin{gathered}
u^{\Delta^{2}}(t)=\nabla F\left(\sigma(t), u^{\sigma}(t)\right), \quad \Delta \text {-a.e. } t \in[0, T]_{\mathbb{T}}^{\kappa^{2}}, \\
u(0)=u(T), \quad u^{\Delta}(0)=u^{\Delta}(T),
\end{gathered}
$$

where $F(t, x)$ is $\Delta$-measurable in $t$ for every $x \in \mathbb{R}^{N}$ and continuously differentiable in $x$ for $\Delta$-a.e. $t \in[0, T]_{\mathrm{T}}$.

Motivated by the works cited previously, in this paper, we study the second order Dirichlet boundary value problem on time scales. Consider

$$
\begin{gathered}
-u^{\Delta \Delta}(t)=\lambda u^{\sigma}(t)+h\left(\sigma(t), u^{\sigma}(t)\right), \quad \Delta \text {-a.e. } t \in J, \\
u(0)=u(T)=0,
\end{gathered}
$$

where we say that a property holds for $\Delta$-almost every $t \in A \subset$ $\mathbb{T}$ or $\Delta$-almost everywhere on $A \subset \mathbb{T}, \Delta$-a.e., whenever there exists a set $E \subset A$ with null Lebesgue $\Delta$-measure such that this property holds for every $t \in A \backslash E, J:=[0, \rho(T)) \cap \mathbb{T}$ and $\mathbb{T} \subset \mathbb{R}$ is an arbitrary bounded time scale such that $\min \mathbb{T}=0$ and $\max \mathbb{T}=T$.

We assume that $\lambda$ is a parameter, $h \in \operatorname{CAR}\left([0, T)_{\mathbb{T}}, \mathbb{R}\right)$, $[0, T)_{\mathbb{T}}:=[0, T) \cap \mathbb{T}$, where $\operatorname{CAR}\left([0, T)_{\mathbb{T}}, \mathbb{R}\right)$ means that $h$ fulfils the Carathéodory conditions (see $[15$, Definition 3.2.22] and [2, (i) and (ii) in $\left.\left(\mathrm{H}_{2}\right)\right]$ ). By virtue of the LeraySchauder degree theory, a result involving the existence of weak solutions is established. Next, we utilize two critical point theorems to investigate that problem (3) has at least one weak solution and infinitely many weak solutions with the parameter $\lambda<0$, respectively. The results obtained here improve some existing results in the literature. 


\section{Preliminaries and an Existence Theorem via Leray-Schauder Degree Theory}

We first offer some related preliminaries concerning the basic definitions and results on time scales. For convenience, for $f \in L_{\Delta}^{2}\left([0, T)_{\mathbb{T}}, \mathbb{R}\right)$, we denote $\int_{0}^{T}|f(s)|^{2} \Delta s=$ $\int_{[0, T)_{\pi}}|f(s)|^{2} \Delta s$. Let Sobolev's space $W_{\Delta, T}^{1,2}$ be defined by (see $[1-3])$

$$
\begin{gathered}
W_{\Delta, T}^{1,2}:=\left\{x \in A C\left([0, T]_{\mathbb{T}}, \mathbb{R}\right): x^{\Delta} \in L_{\Delta}^{2}\left([0, T)_{\mathbb{T}}, \mathbb{R}\right),\right. \\
x(0)=x(T)=0\},
\end{gathered}
$$

where $A C\left([0, T]_{\mathbb{T}}, \mathbb{R}\right)$ (see $[3$, Definition 2.9]) denotes the set of absolutely continuous functions on $[0, T]_{\mathbb{T}}$. Then $W_{\Delta, T}^{1,2}$ is a Hilbert space with the inner product

$$
(u, v)_{1}=\int_{[0, T)_{\mathbb{T}}} u^{\Delta}(t) v^{\Delta}(t) \Delta t,
$$

and let $\|\cdot\|_{1}$ be the norm induced by the inner product $(\cdot, \cdot)_{1}$ (see [1, page 1265] and [2, page 371]).

Lemma 1 (see [3, Proposition 3.7]). The immersion $W_{\Delta, T}^{1,2} \hookrightarrow$ $C\left([0, T]_{\mathbb{T}}, \mathbb{R}\right)$ is compact. lem:

We firstly need to consider the following eigenvalue prob-

$$
\begin{gathered}
-u^{\Delta \Delta}(t)=\lambda u^{\sigma}(t), \quad t \in[0, T]_{\mathbb{T}}, \\
u(0)=u(T)=0 .
\end{gathered}
$$

For each $v \in W_{\Delta, T}^{1,2}$, multiply by $v^{\sigma}(t)$ on both sides of the previous equation in (6) and integrate over $[0, T)_{\mathbb{T}}$ to obtain

$$
\int_{[0, T)_{\mathbb{T}}}-u^{\Delta \Delta}(t) v^{\sigma}(t) \Delta t=\lambda \int_{[0, T)_{\mathbb{T}}} u^{\sigma}(t) v^{\sigma}(t) \Delta t .
$$

By (7) of [8], we obtain

$$
\int_{[0, T)_{\mathbb{T}}} u^{\Delta}(t) v^{\Delta}(t) \Delta t=\lambda \int_{[0, T)_{\mathbb{T}}} u^{\sigma}(t) v^{\sigma}(t) \Delta t .
$$

Lemma 2 (see [9, Lemma 3.4] and [13, Theorem 4.95]). The eigenvalues of (6) may be arranged as $0<\lambda_{1}<\lambda_{2}<\cdots$, and $\lambda_{1}$ can be expressed by

$$
\lambda_{1}:=\inf _{u \in W_{\Delta, T}^{1,2}, u \neq 0} \frac{\int_{[0, T)_{\mathbb{T}}}\left|u^{\Delta}(t)\right|^{2} \Delta t}{\int_{[0, T)_{\mathbb{T}}}\left|u^{\sigma}(t)\right|^{2} \Delta t} .
$$

By Lemma 2, if there exists $u_{1} \in W_{\Delta, T}^{1,2}$ and $\left\|u_{1}\right\|_{1}=1$, then we have

$$
\frac{1}{\lambda_{1}}=\int_{[0, T)_{\mathbb{T}}}\left|u_{1}^{\sigma}(t)\right|^{2} \Delta t .
$$

Choosing $\varphi_{1}^{\sigma}=\sqrt{\lambda_{1}} u_{1}^{\sigma}$, we can easily obtain

$$
\begin{gathered}
\int_{[0, T)_{\mathbb{T}}}\left|\varphi_{1}^{\sigma}(t)\right|^{2} \Delta t=\lambda_{1} \int_{[0, T)_{\mathbb{T}}}\left|u_{1}^{\sigma}(t)\right|^{2} \Delta t=1, \\
\lambda_{1}=\int_{[0, T)_{\mathbb{T}}}\left|\varphi_{1}^{\Delta}(t)\right|^{2} \Delta t .
\end{gathered}
$$

Moreover, we also have

$$
\begin{array}{r}
\int_{[0, T)_{\mathbb{T}}} \varphi_{1}^{\Delta}(t) v^{\Delta}(t) \Delta t=\lambda_{1} \int_{[0, T)_{\mathbb{T}}} \varphi_{1}^{\sigma}(t) v^{\sigma}(t) \Delta t, \\
\forall v \in W_{\Delta, T}^{1,2} .
\end{array}
$$

Lemma 3 (see [16, Corollary 3.3]). Let $u \in W_{\Delta, T}^{1,2}$. Then the Wirtinger-type inequality is

$$
\int_{[0, T)_{\mathbb{T}}}\left|u^{\sigma}(t)\right|^{2} \Delta t \leq \frac{1}{\lambda_{1}} \int_{[0, T)_{\mathbb{T}}}\left|u^{\Delta}(t)\right|^{2} \Delta t,
$$

where $\lambda_{1}$ is defined by Lemma 2.

Denote an operator $A: W_{\Delta, T}^{1,2} \rightarrow W_{\Delta, T}^{1,2}$ as follows

$$
(A u, v)=\int_{[0, T)_{\mathbb{T}}} u^{\sigma}(t) v^{\sigma}(t) \Delta t, \quad \forall u, v \in W_{\Delta, T}^{1,2}
$$

By Hölders inequality and Lemma 3, we have

$$
\begin{aligned}
& \left|\int_{[0, T)_{\mathbb{T}}} u^{\sigma}(t) v^{\sigma}(t) \Delta t\right| \\
& \quad \leq \int_{[0, T)_{\mathbb{T}}}\left|u^{\sigma}(t) v^{\sigma}(t)\right| \Delta t \\
& \quad \leq\left(\int_{[0, T)_{\mathbb{T}}}\left|u^{\sigma}(t)\right|^{2} \Delta t\right)^{1 / 2}\left(\int_{[0, T)_{\mathbb{T}}}\left|v^{\sigma}(t)\right|^{2} \Delta t\right)^{1 / 2} \\
& \leq \frac{1}{\lambda_{1}}\|u\|_{1}\|v\|_{1}, \quad \forall u, v \in W_{\Delta, T}^{1,2} .
\end{aligned}
$$

Then $A$ is a bounded linear operator. By Lemma 1, $A$ is compact. Clearly, $A$ is also symmetric, $(A u, u)>0$ for $u^{\sigma} \neq \equiv$. Consequently, the supremum $1 / \lambda_{1}$ is achieved by Theorem 6.3 .12 in [15]. Hence, (10)-(12) are true.

In what follows, we offer two lemmas involving LeraySchauder degree.

Lemma 4 (see [15, Proposition 5.2.22]). Let $\Omega$ be an open bounded set in a Banach space $X$ and $A \in \mathscr{C}(\bar{\Omega}, X)$. Let $x_{0} \in \Omega$ be a unique solution in $\bar{\Omega}$ of the equation $x=F(x)$. Assume that the Fréchet derivative $A^{\prime}\left(x_{0}\right)$ exists and $I-A^{\prime}\left(x_{0}\right)$ is continuously invertible. Then

$$
\begin{aligned}
& \operatorname{deg}(I-A, \Omega, 0)=(-1)^{\beta}, \\
& \text { where } \beta=\sum_{\lambda \in \sigma\left(F^{\prime}\left(x_{0}\right)\right) \cap \mathbb{R}, \lambda>1} m(\lambda),
\end{aligned}
$$

and $m(\lambda)$ is the multiplicity of the eigenvalue $\lambda$ of the operator $A^{\prime}\left(x_{0}\right)$.

Lemma 5 (see [15, Theorem 5.2.13]). Let $\Omega$ be an open bounded set in a Banach space $X$. There exists a mapping $\operatorname{deg}\left(I-A, \Omega, y_{0}\right)$ defined for all $A \in \mathscr{C}(\bar{\Omega}, X)$ and $y_{0} \in X$ such that $x-A(x) \neq y_{0}, \forall x \in \partial \Omega$. This mapping has the homotopy invariance property: if $A, B \in \mathscr{C}(\bar{\Omega}, X)$ and $H(t, x)=(1-$ t) $A x+t B x, t \in[0,1]$, and $x \in \bar{\Omega}$ are such that $x-H(t, x) \neq y_{0}$, 
for every $x \in \partial \Omega$ and $t \in[0,1]$, then $\operatorname{deg}\left(I-A, \Omega, y_{0}\right)=$ $\operatorname{deg}\left(I-B, \Omega, y_{0}\right)$.

Now, we list our hypotheses for (3).

(H1) $\lambda$ is a parameter and $\lambda \neq \lambda_{n}$, where $\lambda_{n}$ are determined by Lemma 2 .

(H2) $h \in \mathrm{CAR}\left([0, T)_{\mathbb{T}}, \mathbb{R}\right)$; there exist $f \in L_{\Delta}^{2}\left([0, T)_{\mathbb{T}}, \mathbb{R}\right)$, $c>0$ and $\alpha \in[0,1)$ such that

$h(t, x) \leq f(t)+c|x|^{\alpha}, \quad \forall t \in[0, T)_{\mathbb{T}}, x \in \mathbb{R}$.

(H3) $h$ is $\alpha$-Lipschitz continuous with respect to the second variable; that is, there exists a constant $c>0$, such that

$$
\begin{aligned}
& \left|h\left(t, x_{1}\right)-h\left(t, x_{2}\right)\right| \leq c\left|x_{1}-x_{2}\right|^{\alpha}, \\
& \forall t \in[0, T)_{\mathbb{T}}, x_{1}, x_{2} \in \mathbb{R}, \alpha \text { as in (H2). }
\end{aligned}
$$

Remark 6. We can take $h(t, x)=f(t)+c|x|^{\alpha}$, where $c, f, \alpha$ are as in (H2). Clearly, it also satisfies (H3). In fact,

$$
\begin{array}{r}
\left|h\left(t, x_{1}\right)-h\left(t, x_{2}\right)\right|=\left.c|| x_{1}\right|^{\alpha}-\left|x_{2}\right|^{\alpha}|\leq c| x_{1}-\left.x_{2}\right|^{\alpha}, \\
\forall x_{1}, x_{2} \in \mathbb{R} .
\end{array}
$$

It is clear that (3) is equivalent to the following integral equation:

$$
\begin{aligned}
\int_{[0, T)_{\mathbb{T}}} u^{\Delta}(t) v^{\Delta}(t) \Delta t \\
=\lambda \int_{[0, T)_{\mathbb{T}}} u^{\sigma}(t) v^{\sigma}(t) \Delta t \\
\quad+\int_{[0, T)_{\mathbb{T}}} h\left(\sigma(t), u^{\sigma}(t)\right) v^{\sigma}(t) \Delta t, \quad \forall v \in W_{\Delta, T}^{1,2} .
\end{aligned}
$$

We define an operator $S: W_{\Delta, T}^{1,2} \rightarrow W_{\Delta, T}^{1,2}$ as follows:

$$
(S u, v)=\int_{[0, T)_{\mathbb{T}}} h\left(\sigma(t), u^{\sigma}(t)\right) v^{\sigma}(t) \Delta t, \quad \forall u, v \in W_{\Delta, T}^{1,2}
$$

Lemma 7. $S$ is compact on $W_{\Delta, T}^{1,2}$.

Proof. We first prove that there exists a ball $B(0, R) \subset W_{\Delta T}^{1,2}$ $(R>0)$ such that $S$ maps $B(0, R)$ into itself if $R$ is large enough. Indeed, (H2) leads to

$$
\begin{aligned}
\|S u\|_{1} & =\sup _{\|v\|_{1}=1}|S u, v| \\
& =\sup _{\|v\|_{1}=1} \int_{[0, T)_{\mathbb{T}}} h\left(\sigma(t), u^{\sigma}(t)\right) v^{\sigma}(t) \Delta t
\end{aligned}
$$

$$
\begin{aligned}
\leq & \sup _{\|v\|_{1}=1}\left(\int_{[0, T)_{\mathbb{T}}}\left|h\left(\sigma(t), u^{\sigma}(t)\right)\right|^{2} \Delta t\right)^{1 / 2} \\
& \times\left(\int_{[0, T)_{\mathbb{T}}}\left|v^{\sigma}(t)\right|^{2} \Delta t\right)^{1 / 2} \\
\leq & \frac{1}{\sqrt{\lambda_{1}}}\left(\left.\left.\int_{[0, T)_{\mathbb{T}}}\left|f^{\sigma}(t)+c\right| u^{\sigma}(t)\right|^{\alpha}\right|^{2} \Delta t\right)^{1 / 2} \\
\leq & \sqrt{\frac{2}{\lambda_{1}}}\left(\int_{[0, T)_{\mathbb{T}}}\left|f^{\sigma}(t)\right|^{2} \Delta t\right)^{1 / 2} \\
& +\sqrt{\frac{2 c^{2}}{\lambda_{1}}}\left(\int_{[0, T)_{\mathbb{T}}}\left|u^{\sigma}(t)\right|^{2 \alpha} \Delta t\right)^{1 / 2} \\
\leq & \sqrt{\frac{2}{\lambda_{1}}}\left(\int_{[0, T)_{\mathbb{T}}}\left|f^{\sigma}(t)\right|^{2} \Delta t\right)^{1 / 2} \\
& +\sqrt{\frac{2 c^{2} T^{1-\alpha}}{\lambda_{1}^{1+\alpha}}\|u\|_{1}^{\alpha} \cdot} \\
& +\sqrt{\frac{2 c^{2} T^{1-\alpha}}{\lambda_{1}}}\left(\int_{[0, T)_{\mathbb{T}}}\left|u^{\sigma}(t)\right|^{2} \Delta t\right)^{\alpha / 2} \\
\leq & \sqrt{\frac{2}{\lambda_{1}}}\left(\int_{[0, T)_{\mathbb{T}}}\left|f^{\sigma}(t)\right|^{2} \Delta t\right)^{1 / 2}
\end{aligned}
$$

Let $\eta_{1}:=\sqrt{2 / \lambda_{1}}\left(\int_{[0, T)_{\mathbb{T}}}\left|f^{\sigma}(t)\right|^{2} \Delta t\right)^{1 / 2}$, and $\eta_{2} \quad:=$ $\sqrt{2 c^{2} T^{1-\alpha} / \lambda_{1}^{1+\alpha}}$. For any $u \in B(0, R)$,

$$
\|S u\|_{1}<R \text {, provided } \eta_{1}+\eta_{2} R^{\alpha}<R .
$$

Therefore, the above claim is true. Meanwhile, we also arrive at immediately $S$ is uniformly bounded on $B(0, R)$. On the other hand, we shall prove that $S$ is equicontinuous on $B(0, R)$. By (H3) and Hölders inequality, we have

$$
\begin{aligned}
& \left\|S u_{1}-S u_{2}\right\|_{1} \\
& \leq \sup _{\|v\|_{1}=1}\left(\int_{[0, T)_{\mathbb{T}}}\left|h\left(\sigma(t), u_{1}^{\sigma}(t)\right)-h\left(\sigma(t), u_{2}^{\sigma}(t)\right)\right|\left|v^{\sigma}(t)\right| \Delta t\right) \\
& \leq \sup _{\|v\|_{1}=1}\left(\int_{[0, T)_{\mathbb{T}}} c\left|u_{1}^{\sigma}(t)-u_{2}^{\sigma}(t)\right|^{\alpha}\left|v^{\sigma}(t)\right| \Delta t\right) \\
& \leq c \sup _{\|v\|_{1}=1}\left(\int_{[0, T)_{\mathbb{T}}}\left|u_{1}^{\sigma}(t)-u_{2}^{\sigma}(t)\right|^{2} \Delta t\right)^{\alpha / 2} \\
& \quad \times\left(\int_{[0, T)_{\mathbb{T}}}\left|v^{\sigma}(t)\right|^{2 /(2-\alpha)} \Delta t\right)^{(2-\alpha) / 2}
\end{aligned}
$$




$$
\begin{aligned}
& \leq T^{(1-\alpha) /(2-\alpha)} c \sup _{\|\nu\|_{1}=1}\left(\int_{[0, T)_{\mathbb{T}}}\left|u_{1}^{\sigma}(t)-u_{2}^{\sigma}(t)\right|^{2} \Delta t\right)^{\alpha / 2} \\
& \quad \times\left(\int_{[0, T)_{\mathbb{T}}}\left|v^{\sigma}(t)\right|^{2} \Delta t\right)^{1 /(2-\alpha)} \\
& \leq \frac{T^{(1-\alpha) /(2-\alpha)} c}{\lambda_{1}^{1 /(2-\alpha)+\alpha / 2}}\left\|u_{1}-u_{2}\right\|_{1}^{\alpha} .
\end{aligned}
$$

We have from Arzelà-Ascoli theorem $S$ is compact on $B(0, R) \subset W_{\Delta, T}^{1,2}$.

Theorem 8. Assume that (H1)-(H3) hold; problem (3) has at least a weak solution.

Proof. We will utilize Leray-Schauder degree to prove the result. One is invited to verify that the existence of a solution of (3) is equivalent to the existence of a solution of the operator equation

$$
u=\lambda A u+S u, \quad \forall u \in W_{\Delta, T}^{1,2}
$$

where $A$ and $S$ are defined by (14) and (21), respectively. For the reason that $A$ is bounded, linear, and compact on $W_{\Delta, T}^{1,2}$, we easily see that Fréchet derivative $(\lambda A)^{\prime}$ exists and $I-(\lambda A)^{\prime}$ is continuously invertible by (H1). Consequently, Lemma 4 implies

$$
\operatorname{deg}(I-\lambda A, B(0, R), 0) \neq 0 \text {. }
$$

So, to complete the proof, we have to find an admissible homotopy connecting $I-\lambda A-S(\cdot)$ and $I-\lambda A$. Define

$$
H(\tau, u)=u-\lambda A u-\tau S u, \quad \tau \in[0,1], u \in W_{\Delta, T}^{1,2} .
$$

We shall prove that there exists $R>0$ such that for all $u \in$ $W_{\Delta, T}^{1,2},\|u\|_{1}=R$, and $\tau \in[0,1]$, we obtain

$$
H(\tau, u) \neq 0
$$

If the claim is false, we can find sequences $\left\{u_{n}\right\} \subset W_{\Delta, T}^{1,2}$ and $\left\{t_{n}\right\} \subset[0,1]$ such that $\left\|u_{n}\right\|_{1} \rightarrow \infty$ and

$$
u_{n}-\lambda A u_{n}-\tau_{n} S u_{n}=0 .
$$

Set $v_{n}:=u_{n} /\left\|u_{n}\right\|_{1}$ and divide (29) by $\left\|u_{n}\right\|_{1}$ to get

$$
v_{n}-\lambda A v_{n}-\tau_{n} \frac{S u_{n}}{\left\|u_{n}\right\|_{1}}=0
$$

This is equivalent to

$$
\begin{aligned}
\int_{[0, T)_{\mathbb{T}}} v_{n}^{\Delta}(t) w^{\Delta}(t) \Delta t= & \lambda \int_{[0, T)_{\mathbb{T}}} v_{n}^{\sigma}(t) w^{\sigma}(t) \Delta t \\
& +\tau_{n} \int_{[0, T)_{\mathbb{T}}} \frac{h\left(\sigma(t), u_{n}^{\sigma}(t)\right)}{\left\|u_{n}\right\|_{1}} w^{\sigma}(t) \Delta t,
\end{aligned}
$$

for each $w \in W_{\Delta, T}^{1,2}$. Now, passing to suitable subsequences, without loss of generality, we may assume that $\tau_{n} \rightarrow \tau \in$ $[0,1]$ and $v_{n} \rightarrow v$ in $W_{\Delta, T}^{1,2}$. Note that, similar with (22), we find

$$
\begin{aligned}
\mid \int_{[0, T)_{\mathbb{T}}} & \frac{h\left(\sigma(t), u_{n}^{\sigma}(t)\right)}{\left\|u_{n}\right\|_{1}} w^{\sigma}(t) \Delta t \mid \\
\leq & \int_{[0, T)_{\mathbb{T}}} \frac{\left|h\left(\sigma(t), u_{n}^{\sigma}(t)\right)\right|}{\left\|u_{n}\right\|_{1}}\left|w^{\sigma}(t)\right| \Delta t \\
\leq & \frac{\left(\eta_{1}+\eta_{2}\left\|u_{n}\right\|_{1}^{\alpha}\right)\|w\|_{1}}{\left\|u_{n}\right\|_{1}} \longrightarrow 0, \quad n \rightarrow \infty .
\end{aligned}
$$

On the other hand, by the compactness of $A$, we have $A v_{n} \rightarrow$ $A v$ (see [15, Proposition 2.2.4(iii)]). In (30), let $n \rightarrow \infty$; we have $v=\lambda A v$, and $v \in W_{\Delta, T}^{1,2}$ satisfies $\|v\|_{1}=1$. However, this contradicts our assumption $\lambda \neq \lambda_{n}, n=1,2, \ldots$. This implies that (28) holds. By Lemma 5 and (26), we have

$$
\operatorname{deg}(I-\lambda A-S, B(0, R), 0)=\operatorname{deg}(I-\lambda A, B(0, R), 0) \neq 0 .
$$

Therefore, (3) has at least a weak solution.

\section{Two Existence Theorems via Critical Point Theory}

We still use the Sobolev's space $W_{\Delta, T}^{1,2}$ defined by (4), which is equipped with the inner product

$$
(u, v)_{2}:=\int_{[0, T)_{\mathbb{T}}} u^{\sigma}(t) v^{\sigma}(t) \Delta t+\int_{[0, T)_{\mathbb{T}}} u^{\Delta}(t) v^{\Delta}(t) \Delta t,
$$

and the corresponding norm

$$
\|u\|_{2}:=\left(\int_{[0, T)_{\mathbb{T}}}\left|u^{\sigma}(t)\right|^{2} \Delta t+\int_{[0, T)_{\mathbb{T}}}\left|u^{\Delta}(t)\right|^{2} \Delta t\right)^{1 / 2} .
$$

Now, we will establish the corresponding variational formulations for problem (3) as follows:

$$
\begin{aligned}
\varphi(u)= & \frac{1}{2}\left(\int_{[0, T)_{\mathbb{T}}}\left|u^{\Delta}(t)\right|^{2} \Delta t-\lambda \int_{[0, T)_{\mathbb{T}}}\left|u^{\sigma}(t)\right|^{2} \Delta t\right) \\
& -\int_{[0, T)_{\mathbb{T}}} F\left(\sigma(t), u^{\sigma}(t)\right) \Delta t,
\end{aligned}
$$

where $F(t, \xi)=\int_{0}^{\xi} h(t, s) \mathrm{d} s$.

We now list our hypotheses for (3).

(H4) $F(t, x)$ is $\Delta$-measurable in $t$ for every $x \in \mathbb{R}$ and continuously differentiable in $x$ for $t \in[0, T]_{\mathbb{T}}$, and there exist $\epsilon_{1} \in C\left(\mathbb{R}^{+}, \mathbb{R}^{+}\right)$and $\epsilon_{2} \in L_{\Delta}^{1}\left([0, T]_{\mathbb{T}}, \mathbb{R}^{+}\right)$

$$
|F(t, x)| \leq \epsilon_{1}(|x|) \epsilon_{2}(t), \quad|h(t, x)| \leq \epsilon_{1}(|x|) \epsilon_{2}(t),
$$

for all $x \in \mathbb{R}$ and $\Delta$-a.e. $t \in[0, T]_{\mathbb{T}}$. 
(H5) There exist $c_{1}>0$ and $p>0$ such that

$$
|F(t, x)| \leq c_{1}|x|^{p}, \quad t \in[0, T]_{\mathbb{T}}, u \in \mathbb{R} .
$$

(H6) There exist $\varepsilon_{1}, \varepsilon_{2}>0$ such that

$$
h(t, x) x-2 F(t, x) \geq \varepsilon_{1}|x|-\varepsilon_{2}, \quad t \in[0, T]_{\mathbb{T}}, u \in \mathbb{R} .
$$

(H7) $\lim _{|x| \rightarrow \infty}\left(F(t, x) /|x|^{2}\right)=+\infty$ uniformly for $t \in$ $[0, T]_{\mathbb{T}}$.

(H8) $F(t,-x)=F(t, x), t \in[0, T]_{\mathbb{T}}, u \in \mathbb{R}$.

Remark 9. Let

$$
F(t, x)=\sum_{i=1}^{n} \frac{1}{(2 i)^{2}} x^{2 i}
$$

where $n>1$ is a fixed positive integer. Then

$$
h(t, x)=\sum_{i=1}^{n} \frac{1}{2 i} x^{2 i-1} .
$$

Direct computation shows

$$
\begin{aligned}
& \lim _{|x| \rightarrow \infty} \frac{F(t, x)}{|x|^{2}}=\lim _{|x| \rightarrow \infty} \frac{\sum_{i=1}^{n}\left(1 /(2 i)^{2}\right) x^{2 i}}{|x|^{2}}=+\infty, \\
& \lim _{|x| \rightarrow \infty} \frac{h(t, x) x-2 F(t, x)}{|x|}=\lim _{|x| \rightarrow \infty} \frac{\sum_{i=1}^{n}\left[1 / 2 i-1 / 2 i^{2}\right] x^{2 i}}{|x|} \\
&=+\infty .
\end{aligned}
$$

Clearly, (H4)-(H8) hold.

By (H4), we find $\varphi \in C^{1}\left(W_{\Delta, T}^{1,2}, \mathbb{R}\right)$ (see [11, Theorem 2.27]), and for $u, v \in W_{\Delta, T}^{1,2}$,

$$
\begin{aligned}
\varphi^{\prime}(u) v= & \int_{[0, T)_{\mathbb{T}}} u^{\Delta}(t) v^{\Delta}(t) \Delta t-\lambda \int_{[0, T)_{\mathbb{T}}} u^{\sigma}(t) v^{\sigma}(t) \Delta t \\
& -\int_{[0, T)_{\mathbb{T}}} h\left(\sigma(t), u^{\sigma}(t)\right) v^{\sigma}(t) \Delta t .
\end{aligned}
$$

Clearly, the existence of weak solutions for (3) is equivalent to the existence of critical points for $\varphi$. In what follows, we take $\bar{a}:=\min \{1,-\lambda\}>0, \bar{b}:=\max \{1,-\lambda\}>0$.

Lemma 10 (see [17, Theorem 1.2]). Suppose $X$ is a reflexive Banach space with norm $\|\cdot\|$, and $\varphi: X \rightarrow \mathbb{R} \cup\{+\infty\}$ is coercive and weak (sequentially) lower semicontinuous; that is, the following conditions are fulfilled as follows:

(1) $\varphi(u) \rightarrow \infty$ as $\|u\| \rightarrow \infty, u \in X$.
(2) For any $u \in X$, any sequence $\left\{u_{m}\right\} \subset X$ such that $u_{m} \rightarrow$ $u$, there holds

$$
\varphi(u) \leq \liminf _{m \rightarrow \infty} \varphi\left(u_{m}\right) .
$$

Then $\varphi$ is bounded from below and attains its infimum on $X$.

Theorem 11. If (H4) and (H5) with $p \in(0,2)$ hold, (3) has at least a weak solution.

Proof. Our working space $W_{\Delta, T}^{1,2}$ is a Hilbert space, so it is reflexive. By Lemma 2.1 and Theorem 3.3 in [8], we see $\varphi$ is weakly lower semicontinuous on $W_{\Delta, T}^{1,2}$. On the other hand, by (H5), we have

$$
\begin{aligned}
\varphi(u) & \geq \frac{\bar{a}}{2}\|u\|_{2}^{2}-\int_{[0, T)_{\mathbb{T}}} c_{1}\left|u^{\sigma}(t)\right|^{p} \Delta t \\
& \geq \frac{\bar{a}}{2}\|u\|_{2}^{2}-c_{1} T^{(2-p) / 2}\|u\|_{2}^{p},
\end{aligned}
$$

and thus $\varphi(u) \rightarrow \infty$ as $\|u\|_{2} \rightarrow \infty$. Lemma 10 implies $\varphi$ can attain its infimum in $X$; that is, (3) has at least a weak solution. This completes the proof.

If $X$ is a Hilbert space, there exist (see [18]) $\left\{e_{n}\right\}_{n=1}^{\infty} \subset X$ and $\left\{f_{n}\right\}_{n=1}^{\infty} \subset X^{*}$ such that $f_{n}\left(e_{m}\right)=\delta_{n, m}, X=\overline{\operatorname{span}}\left\{e_{n}: n=\right.$ $1,2, \ldots\}$ and $X^{*}=\overline{\operatorname{span}}^{W^{*}}\left\{f_{n}: n=1,2, \ldots\right\}$. For $j, k \in \mathbb{N}$, denote $X_{j}:=\operatorname{span}\left\{e_{j}\right\}, Y_{k}:=\oplus_{j=1}^{k} X_{j}$, and $Z_{k}:=\overline{\oplus_{j=k+1}^{\infty} X_{j}}$. Clearly, $X=\overline{\oplus_{j \in \mathbb{N}} X_{j}}$ with $\operatorname{dim} X_{j}<\infty$ for all $j \in \mathbb{N}$. Since $W_{\Delta, T}^{1,2}$ is a Hilbert space, we can choose an orthonormal basis $\left\{e_{n}\right\}$ such that

$$
W_{\Delta, T}^{1,2}=\overline{\operatorname{span}}\left\{e_{n}: n=1,2 \ldots\right\}, \quad Y_{k}:=\bigoplus_{j=1}^{k} X_{j},
$$

$$
Z_{k}:=\overline{\bigoplus_{j=k+1}^{\infty} X_{j}}, \quad \text { where } X_{j}:=\operatorname{span}\left\{e_{j}\right\} .
$$

Definition 12 (see [19, Definition 1.1]). Assume that $X$ is a Banach space with norm $\|\cdot\|$; we say that $\varphi \in C^{1}(X, \mathbb{R})$ satisfies Cerami condition (C) if for all $d \in \mathbb{R}$,

(i) any bounded sequence $\left\{u_{n}\right\} \subset X$ satisfying $\varphi\left(u_{n}\right) \rightarrow$ $d, \varphi^{\prime}\left(u_{n}\right) \rightarrow 0$ possesses a convergent subsequence;

(ii) there exist $\delta, \xi, \rho>0$ such that for any $u \in \varphi^{-1}([d-$ $\delta, d+\delta])$ with $\|u\| \geq \xi$, $\left\|\varphi^{\prime}(u)\right\| \cdot\|u\| \geq \rho$. Denote $S_{\rho}:=$ $\{u \in X:\|u\|=\rho\}$. We will introduce the following Fountain theorem under condition (C).

Lemma 13 (see [19, Proposition 1.2]). Assume that $\varphi \in$ $C^{1}(X, \mathbb{R})$ satisfies condition $(C)$, and $\varphi(-u)=\varphi(u)$. For each $k \in \mathbb{N}$, there exists $\rho_{k}>r_{k}>0$ such that

(i) $b_{k}:=\inf _{u \in Z_{k} \cap S_{r_{k}}} \varphi(u) \rightarrow+\infty, k \rightarrow \infty$;

(ii) $a_{k}:=\max _{u \in Y_{k} \cap S_{\rho_{k}}} \varphi(u) \leq 0$. 
Then $\varphi$ has a sequence of critical points $u_{n}$, such that $\varphi\left(u_{n}\right) \rightarrow+\infty$ as $n \rightarrow \infty$.

Lemma 14. Suppose that (H4), (H5), and (H6) hold; then $\varphi$ satisfies the condition (C).

Proof. For all $d \in \mathbb{R}$, we assume that $\left\{u_{n}\right\} \in W_{\Delta, T}^{1,2}$ is bounded and

$$
\varphi\left(u_{n}\right) \longrightarrow d, \quad \varphi^{\prime}\left(u_{n}\right) \longrightarrow 0, \quad n \longrightarrow \infty
$$

Going, if necessary, to a subsequence, we can assume that $u_{n} \rightarrow u$ in $W_{\Delta, T}^{1,2}$; then

$$
\begin{aligned}
& \left(\varphi^{\prime}\left(u_{n}\right)-\varphi^{\prime}(u)\right)\left(u_{n}-u\right) \\
& =\int_{[0, T)_{\mathbb{T}}}\left|u_{n}^{\Delta}(t)-u^{\Delta}(t)\right|^{2} \Delta t-\lambda \int_{[0, T)_{\mathbb{T}}}\left|u_{n}^{\sigma}(t)-u^{\sigma}(t)\right|^{2} \Delta t \\
& -\int_{[0, T)_{\mathbb{T}}}\left[h\left(\sigma(t), u_{n}^{\sigma}(t)\right)-h\left(\sigma(t), u^{\sigma}(t)\right)\right] \\
& \times\left[u_{n}^{\sigma}(t)-u^{\sigma}(t)\right] \Delta t .
\end{aligned}
$$

Lemma 1 leads to

$$
\begin{aligned}
\int_{[0, T)_{\mathbb{T}}} & {\left[h\left(\sigma(t), u_{n}^{\sigma}(t)\right)-h\left(\sigma(t), u^{\sigma}(t)\right)\right] } \\
& \times\left[u_{n}^{\sigma}(t)-u^{\sigma}(t)\right] \Delta t \longrightarrow 0, \quad n \longrightarrow \infty .
\end{aligned}
$$

It follows that $u_{n} \rightarrow u$ in $W_{\Delta, T}^{1,2}$ and $\left(\varphi^{\prime}\left(u_{n}\right)-\varphi^{\prime}(u)\right)\left(u_{n}-u\right) \rightarrow$ 0 , we see

$$
\begin{aligned}
& \int_{[0, T)_{\mathbb{T}}}\left|u_{n}^{\Delta}(t)-u^{\Delta}(t)\right|^{2} \Delta t \\
& \quad-\lambda \int_{[0, T)_{\mathbb{T}}}\left|u_{n}^{\sigma}(t)-u^{\sigma}(t)\right|^{2} \Delta t \longrightarrow 0, \quad n \longrightarrow \infty .
\end{aligned}
$$

Clearly, it is equivalent to $\|\cdot\|_{2}$, and then we have

$$
\left\|u_{n}-u\right\|_{2} \longrightarrow 0, \quad n \longrightarrow \infty
$$

Hence, condition (i) of Definition 12 holds. Next, we prove condition (ii) of Definition 12, suppose the contrary, there exists a sequence $\left\{u_{n}\right\} \subset W_{\Delta, T}^{1,2}$ such that

$$
\begin{gathered}
\varphi\left(u_{n}\right) \longrightarrow d, \quad\left\|\varphi^{\prime}\left(u_{n}\right)\right\|_{2} \cdot\left\|u_{n}\right\|_{2} \longrightarrow 0, \quad n \longrightarrow \infty \\
\left\|u_{n}\right\|_{2} \longrightarrow \infty, \quad n \longrightarrow \infty
\end{gathered}
$$

By (52), there exists a constant $\varepsilon_{3}>0$ such that

$$
\varphi\left(u_{n}\right)-\frac{1}{2} \varphi^{\prime}\left(u_{n}\right) u_{n} \leq c_{3}
$$

On the other hand, (H6) implies

$$
\begin{aligned}
\varphi( & \left.u_{n}\right)-\frac{1}{2} \varphi^{\prime}\left(u_{n}\right) u_{n} \\
& =\int_{[0, T)_{\mathbb{T}}}\left[\frac{1}{2} h\left(\sigma(t), u_{n}^{\sigma}(t)\right) u_{n}^{\sigma}(t)-F\left(\sigma(t), u_{n}^{\sigma}(t)\right)\right] \Delta t \\
& \geq \frac{1}{2} \varepsilon_{1} \int_{[0, T)_{\mathbb{T}}}\left|u_{n}^{\sigma}(t)\right| \Delta t-\frac{1}{2} \varepsilon_{2} T .
\end{aligned}
$$

This, together with (54), leads to there is a constant $\varepsilon_{4}>0$ such that $\max _{t \in[0, T]_{\pi}}\left|u_{n}^{\sigma}(t)\right| \leq \varepsilon_{4}$. By (H5), similar with (45), we have

$$
\varphi\left(u_{n}\right) \geq \frac{\bar{a}}{2}\left\|u_{n}\right\|_{2}^{2}-\int_{[0, T)_{\mathbb{T}}} c_{1}\left|u_{n}^{\sigma}(t)\right|^{p} \Delta t \geq \frac{\bar{a}}{2}\left\|u_{n}\right\|_{2}^{2}-c_{1} T \varepsilon_{4}^{p},
$$

and thus $\varphi\left(u_{n}\right) \rightarrow \infty$ if (53) holds, which contradicts $\varphi\left(u_{n}\right) \rightarrow d$ in (52). This proves that $\varphi$ satisfies condition (C).

Theorem 15. Under assumptions (H4)-(H8) with $p>2$ in (H5), problem (3) has infinitely many solutions.

Proof. (H8) and Lemma 14 enable us to obtain that $\varphi(u)=$ $\varphi(-u)$ and $\varphi$ satisfies the condition (C). For any $u \in Y_{k}$, let

$$
\|u\|_{*}:=\left(\int_{[0, T)_{\mathbb{T}}}\left|u^{\sigma}(t)\right|^{2} \Delta t\right)^{1 / 2}
$$

and it is easy to verify that $\|\cdot\|$ defined by (57) is a norm of $Y_{k}$. Since all the norms of a finite dimensional normed space are equivalent, so there exists positive constant $\varepsilon_{5}$ such that $\varepsilon_{5}\|u\|_{2} \leq\|u\|_{*}$. In view of (H7), there exist $\varepsilon_{6}>\bar{b} / 2 \varepsilon_{5}^{2}$ and $\varrho>0$ such that

$$
F\left(\sigma(t), u^{\sigma}(t)\right) \geq \varepsilon_{6}\left|u^{\sigma}(t)\right|^{2}-\varrho, \quad t \in[0, T]_{\mathbb{T}}, u \in \mathbb{R} .
$$

This implies that

$$
\begin{aligned}
\varphi(u)= & \frac{1}{2}\left(\int_{[0, T)_{\mathbb{T}}}\left|u^{\Delta}(t)\right|^{2} \Delta t-\lambda \int_{[0, T)_{\mathbb{T}}}\left|u^{\sigma}(t)\right|^{2} \Delta t\right) \\
& -\int_{[0, T)_{\mathbb{T}}} F\left(\sigma(t), u^{\sigma}(t)\right) \Delta t \\
\leq & \frac{\bar{b}}{2}\|u\|_{2}^{2}-\varepsilon_{6} \varepsilon_{5}^{2}\|u\|_{2}^{2}+\varrho T .
\end{aligned}
$$

Since $\bar{b} / 2-\varepsilon_{6} \varepsilon_{5}^{2}<0$; then there exists positive constant $d_{k}$ such that

$$
\varphi(u) \leq 0, \quad \text { for each } u \in Y_{k},\|u\| \geq d_{k} \text {. }
$$


Let $\beta_{k}:=\sup _{u \in Z_{k},\|u\|_{2}=1}|u(t)|, t \in[0, T]_{\mathbb{T}}$. Then by Lemma 3.8 of [20] and Lemma 1 we obtain $\beta_{k} \rightarrow 0$, as $k \rightarrow \infty$. For any $u \in Z_{k}$, note that $p \in(1,2)$, in view of $(\mathrm{H} 5)$, we find that

$$
\begin{aligned}
\varphi(u)= & \frac{1}{2}\left(\int_{[0, T)_{\top}}\left|u^{\Delta}(t)\right|^{2} \Delta t-\lambda \int_{[0, T)_{\top}}\left|u^{\sigma}(t)\right|^{2} \Delta t\right) \\
& -\int_{[0, T)_{\top}} F\left(\sigma(t), u^{\sigma}(t)\right) \Delta t \\
\geq & \frac{\bar{a}}{2}\|u\|_{2}^{2}-c_{1} \int_{[0, T)_{\top}}\left|u^{\sigma}(t)\right|^{p} \Delta t \\
\geq & \frac{\bar{a}}{2}\|u\|_{2}^{2}-c_{1} T\|u\|_{\infty}^{p} \\
\geq & \frac{\bar{a}}{2}\|u\|_{2}^{2}-c_{1} T \beta_{k}^{p}\|u\|_{2}^{p} .
\end{aligned}
$$

Choosing $\|u\|_{2}=r_{k}:=1 / \beta_{k}$, then $r_{k} \rightarrow \infty$ as $k \rightarrow \infty$, then we have;

$$
\varphi(u) \geq \frac{\bar{a}}{2} r_{k}^{2}-c_{1} T \longrightarrow \infty, \quad \text { as } k \longrightarrow \infty .
$$

Hence, $b_{k}:=\inf _{u \in Z_{k},\|u\|=r_{k}} \varphi(u) \rightarrow \infty$ as $k \rightarrow \infty$. Combining this and (60), we can take $\rho_{k}:=\max \left\{d_{k}, r_{k}+1\right\}$, and thus $a_{k}:=\max _{u \in Y_{k},\|u\|=\rho_{k}} \varphi(u) \leq 0$. Up until now, we have proved that the functional $\varphi$ satisfies all the conditions of Lemma 13; then $\varphi$ has infinitely many solutions.

\section{Acknowledgment}

This research is supported by the Project of Shandong Province Higher Educational Science and Technology Program (Grant no. J13LI51).

\section{References}

[1] R. P. Agarwal, V. Otero-Espinar, K. Perera, and D. R. Vivero, "Existence of multiple positive solutions for second order nonlinear dynamic BVPs by variational methods," Journal of Mathematical Analysis and Applications, vol. 331, no. 2, pp. 1263-1274, 2007.

[2] R. P. Agarwal, V. Otero-Espinar, K. Perera, and D. R. Vivero, "Multiple positive solutions of singular Dirichlet problems on time scales via variational methods," Nonlinear Analysis A: Theory, Methods \& Applications, vol. 67, no. 2, pp. 368-381, 2007.

[3] R. P. Agarwal, V. Otero-Espinar, K. Perera, and D. R. Vivero, "Basic properties of Sobolev's spaces on time scales," Advances in Difference Equations, vol. 2006, Article ID 038121, 2006.

[4] V. Otero-Espinar and T. Pernas-Castaño, "Variational approach to second-order impulsive dynamic equations on time scales," Boundary Value Problems, vol. 2013, article 119, 2013.

[5] Y.-H. Su and W.-T. Li, "Periodic solution for non-autonomous second order Hamiltonian systems on time scales," Dynamic Systems and Applications, vol. 18, no. 3-4, pp. 621-636, 2009.

[6] Y.-H. Su and W.-T. Li, "Periodic solution of second-order Hamiltonian systems with a change sign potential on time scales," Discrete Dynamics in Nature and Society, vol. 2009, Article ID 328479, 17 pages, 2009.
[7] Y.-H. Su and Z. Feng, "A non-autonomous Hamiltonian system on time scales," Nonlinear Analysis A: Theory, Methods \& Applications, vol. 75, no. 10, pp. 4126-4136, 2012.

[8] J. Zhou and Y. Li, "Sobolev's spaces on time scales and its applications to a class of second order Hamiltonian systems on time scales," Nonlinear Analysis A: Theory, Methods \& Applications, vol. 73, no. 5, pp. 1375-1388, 2010.

[9] Q.-G. Zhang and H.-R. Sun, "Variational approach for SturmLiouville boundary value problems on time scales," Journal of Applied Mathematics and Computing, vol. 36, no. 1-2, pp. 219232, 2011.

[10] J. Zhou and Y. Li, "Variational approach to a class of second order Hamiltonian systems on time scales," Acta Applicandae Mathematicae, vol. 117, pp. 47-69, 2012.

[11] Y. Li and J. Zhou, "Existence of solutions for a class of damped vibration problems on time scales," Advances in Difference Equations, Article ID 727486, 27 pages, 2010.

[12] Q. Zhang, X. He, and H. Sun, "Positive solutions for SturmLiouville BVPs on time scales via sub- supersolution and variational methods," Boundary Value Problems, vol. 2013, article 123, 2013.

[13] M. Bohner and A. Peterson, Dynamic Equations on Time Scales: An Introduction with Applications, Birkhäuser Boston, Boston, Mass, USA, 2001.

[14] M. Bohner and A. Peterson, Advances in Dynamic Equations on Time Scales, Applications to Differential Equations, Birkhäuser Boston, Boston, Mass, USA, 2003.

[15] P. Drábek and J. Milota, Methods of Nonlinear Analysis, Birkhäuser, Basel, Switzerland, 2007.

[16] R. P. Agarwal, V. Otero-Espinar, K. Perera, and D. R. Vivero, "Wirtinger's inequalities on time scales," Canadian Mathematical Bulletin, vol. 51, no. 2, pp. 161-171, 2008.

[17] M. Struwe, Variational Methods: Applications to Nonlinear Partial Differential Equations and Hamiltonian Systems, vol. 34, Springer, Berlin, Germany, 4th edition, 2008.

[18] X. Fan and X. Han, "Existence and multiplicity of solutions for $\mathbb{R}^{N}$-Laplacian equations in $R^{N}$," Nonlinear Analysis A: Theory, Methods \& Applications, vol. 59, no. 1-2, pp. 173-188, 2004.

[19] A. Qian and C. Li, "Infinitely many solutions for a Robin boundary value problem," International Journal of Differential Equations, Article ID 548702, 9 pages, 2010.

[20] M. Willem, Minimax Theorems, Birkhäuser Boston, Boston, Mass, USA, 1996. 


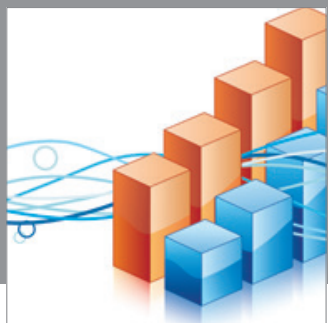

Advances in

Operations Research

mansans

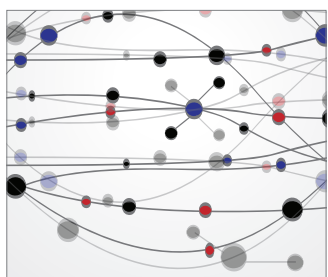

The Scientific World Journal
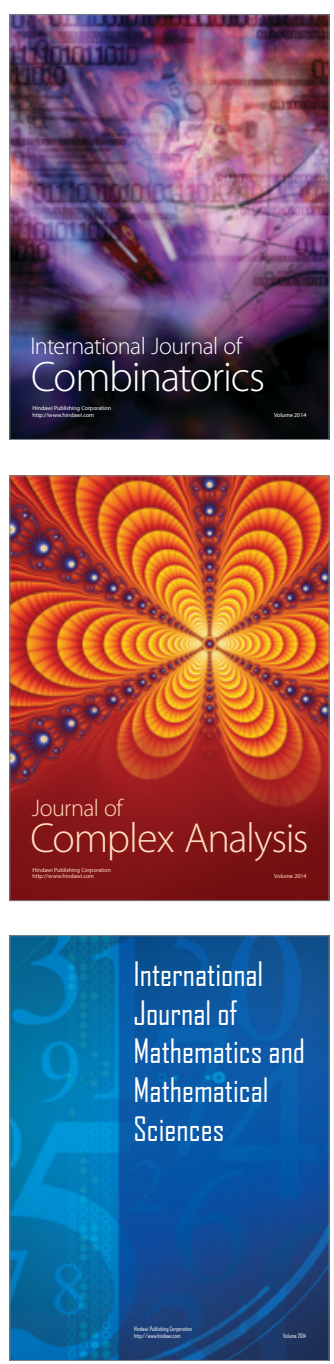
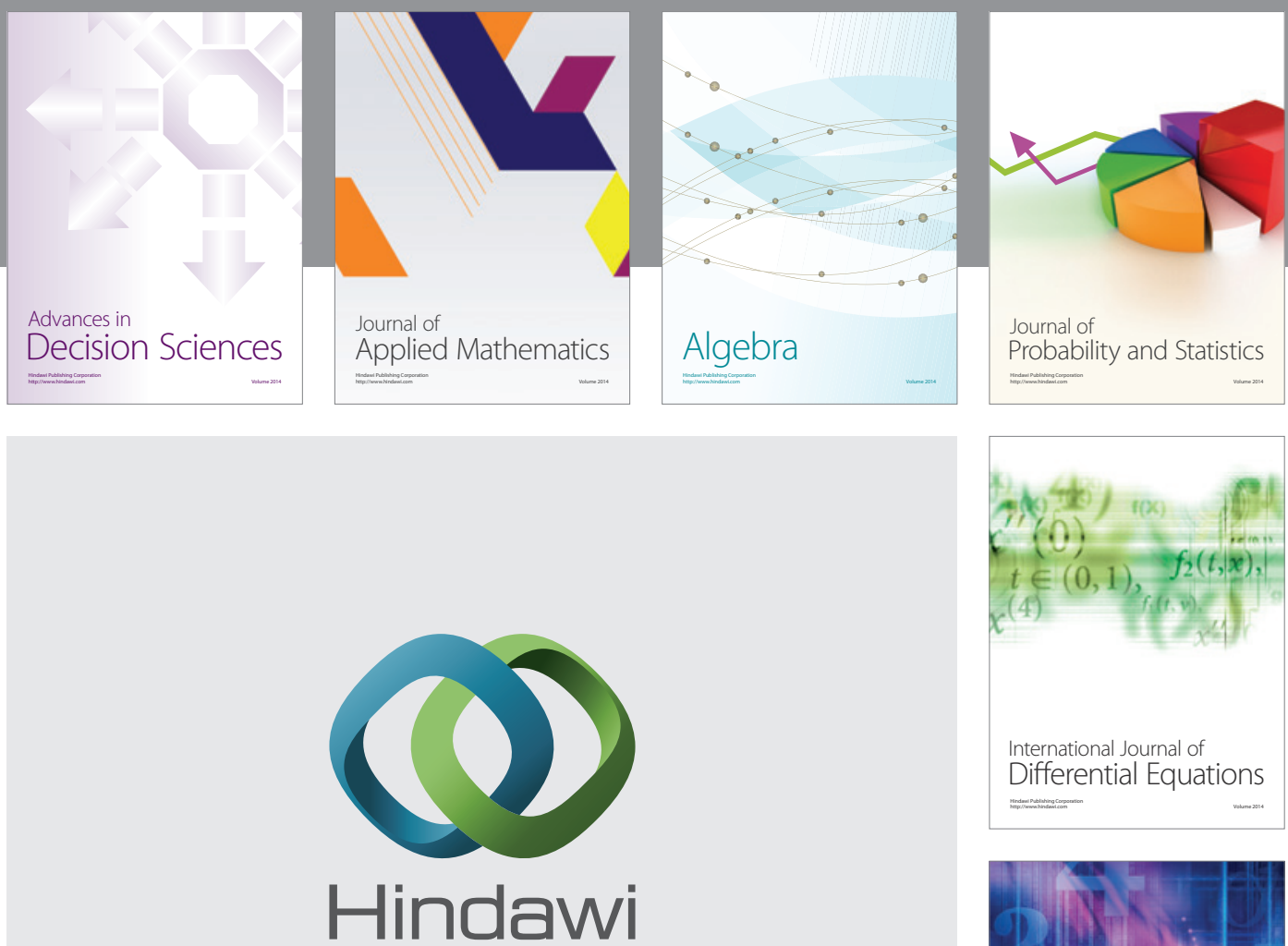

Submit your manuscripts at http://www.hindawi.com
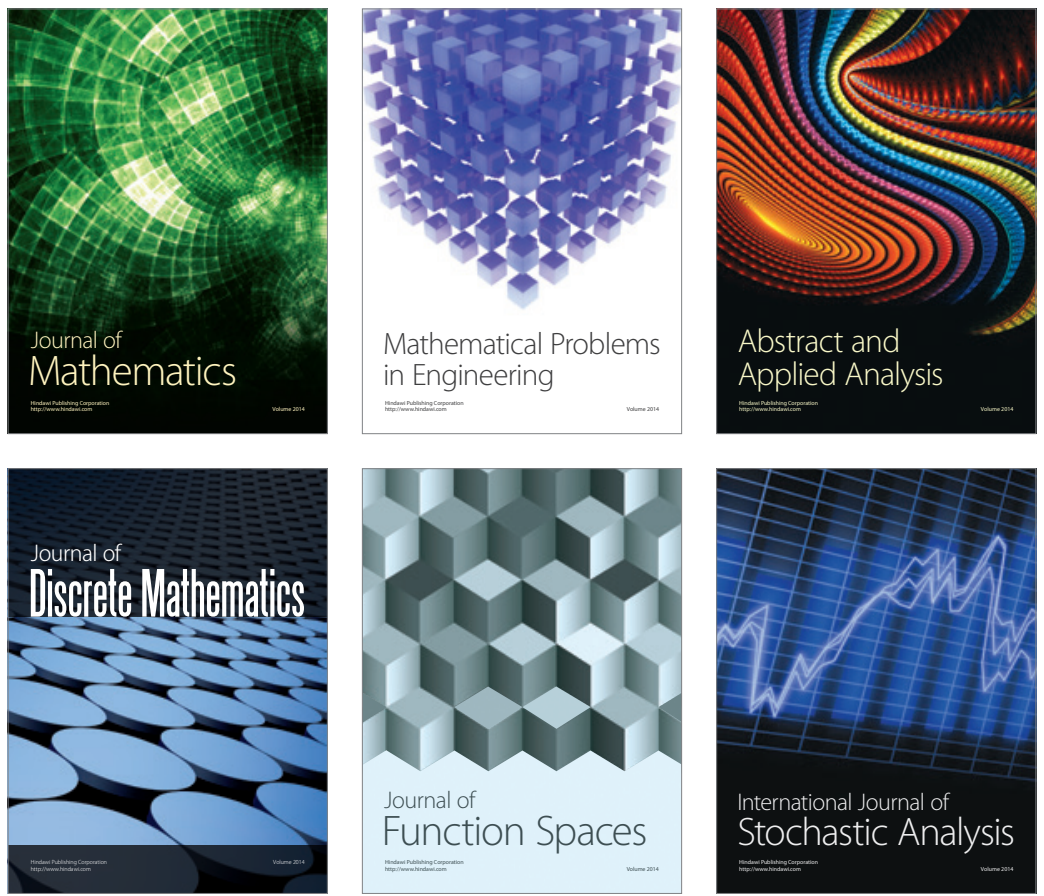

Journal of

Function Spaces

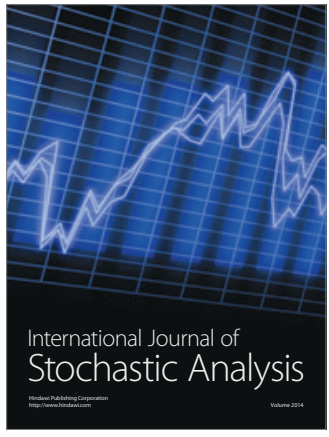

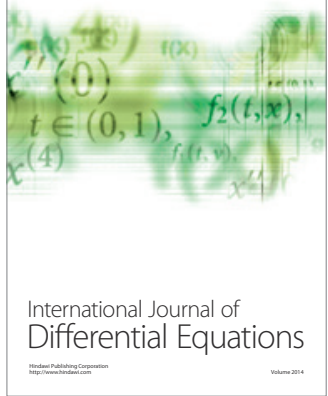
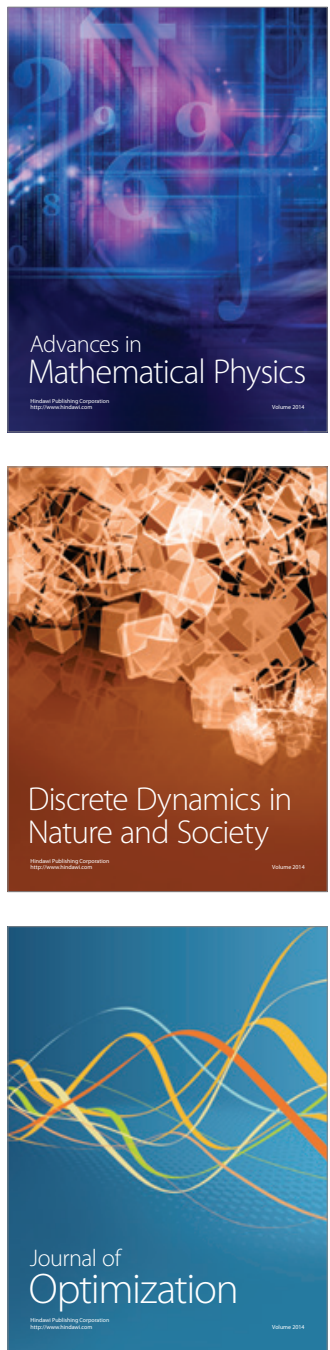